\title{
Health literacy in a population-based sample of Australian women: a cross-sectional profile of the Geelong Osteoporosis Study
}

\author{
Sarah M. Hosking ${ }^{1,2,3^{*}}$ (D), Sharon L. Brennan-Olsen ${ }^{1,2,4,5,6}$, Alison Beauchamp ${ }^{2,7}$, Rachelle Buchbinder ${ }^{8,9}$,
} Lana J. Williams ${ }^{1}$ and Julie A. Pasco $1,5,8$

\begin{abstract}
Background: The term health literacy refers to the abilities and resources required to find, understand and use health information in managing health. This definition is reflected in the recent development of multidimensional health literacy tools that measure multiple facets of health literacy. The aim of this study was to determine the health literacy profile of a randomly selected, population-based sample of Australian women using a multidimensional tool, the Health Literacy Questionnaire (HLQ). A second aim was to investigate associations between independent HLQ scales, sociodemographic characteristics and lifestyle and anthropometric risk factors for chronic disease.

Methods: We surveyed women involved in the Geelong Osteoporosis Study (GOS), a longitudinal, population-based study. We included demographic data, lifestyle information and anthropometric measures as well as the HLQ. The HLQ has 44 items, scored on either 4- or 5-point scales, within nine conceptually distinct scales. Means for each scale were calculated, and HLQ scales were regressed on educational level and socioeconomic status. Risk factors for chronic disease were investigated using analysis of variance (ANOVA) and calculation of effect sizes.

Results: Higher mean scores were seen for the scales 'Feeling understood and supported by healthcare professionals' (mean 3.20, \pm SD 0.52) and 'Understanding health information well enough to know what to do' (mean 4.28, \pm SD 0.54), and lower mean scores were seen for 'Appraisal of health information' (mean 2.81, \pm SD 0.48) and 'Navigating the healthcare system' (mean 4.09, \pm SD 0.57). Associations were also seen between lower HLQ scores and poor health behaviours including smoking and being more sedentary, in addition to greater body mass index and waist circumference. Positive gradients were seen between several HLQ scales and education level, as well as SES. For some HLQ scales, these associations were non-linear.

Conclusions: The profile of this population-based cohort of women demonstrated associations between low health literacy and low SES, lower levels of education, increasing age, and anthropometric and lifestyle risk factors for chronic disease. These findings suggest implications of health literacy for health policy makers focusing on improving lifestyle prevention of chronic disease and promoting health equity at a population level.
\end{abstract}

Keywords: Health literacy, Chronic disease, Health inequities, Sociodemographic characteristics

\footnotetext{
* Correspondence: SHOSKI@BarwonHealth.org.au

${ }^{1}$ Epi-Centre for Healthy Ageing, School of Medicine, IMPACT SRC, Deakin

University, PO Box 281 (Barwon Health), Geelong, VIC 3220, Australia

${ }^{2}$ Australian Institute for Musculoskeletal Science (AIMSS), The University of

Melbourne and Western Health, St Albans, VIC, Australia

Full list of author information is available at the end of the article
}

(c) The Author(s). 2018 Open Access This article is distributed under the terms of the Creative Commons Attribution 4.0 International License (http://creativecommons.org/licenses/by/4.0/), which permits unrestricted use, distribution, and reproduction in any medium, provided you give appropriate credit to the original author(s) and the source, provide a link to the Creative Commons license, and indicate if changes were made. The Creative Commons Public Domain Dedication waiver (http://creativecommons.org/publicdomain/zero/1.0/) applies to the data made available in this article, unless otherwise stated. 


\section{Background}

Health literacy is defined by the World Health Organization (WHO) as 'the cognitive and social skills which determine the motivation and ability of individuals to gain access to, understand and use information in ways which promote and maintain good health' [1].

Previous research has estimated that $59 \%$ of Australian adults do not have adequate health literacy skills to manage their health [2]. This figure is comparable to similar high-income countries including Canada and New Zealand [3]. Within the general population there are groups more likely to report low health literacy. These include culturally and linguistically diverse (CALD) populations, individuals with lower income or education level and older adults [4-6] There is emerging evidence to suggest that low health literacy may be a mediator in the relationship between social disadvantage and poor health behaviours and outcomes [7, 8].

While the relationship between low health literacy and poorer management of chronic disease has previously been established [9-11], evidence suggests low health literacy may also influence lifestyle prevention of chronic disease. Associations between higher health literacy and some lifestyle behaviours including healthier diet and increased physical activity have been demonstrated [12-17]. However, associations between health literacy and smoking and alcohol intake have been inconsistent $[12,16,18,19]$, potentially due to the variation between study populations and health literacy measures used.

Prevention and management of chronic disease is complex and requires a broad range of health literacy abilities and supports. However, the majority of previous health literacy research has investigated only a narrow set of basic literacy and numeracy skills applied to health [20-23]. More recently, a number of multidimensional tools have been developed that enable researchers to investigate a range of abilities and contextual factors associated with health literacy [24-27]. To date, research that has employed multidimensional measures of health literacy has focused on specific populations such as university students [28, 29], recently hospitalised individuals $[4,5]$ and patients groups $[13,30]$. There is comparatively little data investigating health literacy in the broader population.

Further research is required to understand the broad range of health literacy abilities and supports in the wider population and the role they play in the uptake of lifestyle recommendations for the prevention of chronic disease. This information would assist in informing public policy, allocating resources and developing interventions to address low health literacy and reduce health inequalities at a population level [31].

The current study aims to address gaps in the literature using a multidimensional health literacy measure, the Health Literacy Questionnaire (HLQ), in a population-based sample of women. The HLQ is a multidimensional tool developed in Australia using a grounded approach [24]. Preliminary work has shown the HLQ has acceptable measurement properties and measures health literacy across nine distinct domains [24, 32]. The HLQ is a widely used measure of health literacy, having been translated and used in many countries across the world [4-6, 29, 33]. Aside from one Danish study, which included two of the nine scales that make up the HLQ to assess health literacy in the general population [6], the full HLQ has not yet been applied to the general population.

The aim of this study was to describe the health literacy profile of a randomly recruited population-based sample of women participating in a cohort study based in south-eastern Australia. A second aim was to investigate whether there were any associations between HLQ scales and socio-demographic characteristics and risk factors for chronic disease.

\section{Method \\ Participants}

Data were collected from women participating in the prospective, population-based Geelong Osteoporosis Study (GOS); the GOS protocol has been published elsewhere [34]. In brief, a cohort of 1494 women was randomly recruited from the general population between 1993 and 1997 (77.1\% participation) with a further 246 women aged 20-29 years recruited at the same time as the 10 -year follow up. All participants enrolled in the GOS in December 2014 were sent the HLQ to complete, with data collection continuing until March 2016. All participants gave written, informed consent to be involved in the GOS. The Barwon Health Human Research Ethics Committee approved the study.

\section{Data collection}

Participants completed the HLQ online or via post. Participants who required assistance in completing the questions were given the opportunity to have a friend or relative assist as well as being offered the option of completing the questionnaire over the phone with a member of the research team. A question within the HLQ itself captured information regarding whether or not participants had been assisted in completing the questionnaire and, if so, in what way they were assisted. Electronic data were collected via the Research Electronic Data Capture (REDCap) tool [35] hosted by Barwon Health, which was also used to enter and manage hard copy questionnaires.

\section{Measure}

The HLQ is a 44-item, multidimensional tool that determines health literacy scores across nine conceptually distinct domains, each measured by an independent scale. 
Previous research has determined the nine scales of the HLQ measure separate health literacy constructs and have good internal consistency and reliability [24, 32].

The nine scales that comprise the HLQ are:

1. Feeling understood and supported by healthcare providers

2. Having sufficient information to manage my health

3. Actively managing my health

4. Social support for health

5. Appraisal of health information

6. Ability to actively engage with healthcare providers

7. Navigating the healthcare system

8. Ability to find good health information

9. Understand health information well enough to know what to do [24].

Each scale includes between four and six items. Scales 1-5 encompass items scored on a 4-point scale (strongly disagree, disagree, agree, and strongly agree) and reflect an individual's supports, motivation and confidence in managing their health. Scales 6-9 are scored on a 5-point scale (cannot do, very difficult, quite difficult, easy, and very easy) and broadly capture an individual's capability to engage with, and use health information and health services, often based on lived experiences [24].

Data, including education level, health conditions, current smoking, possession of a healthcare card (a concession card available to individuals on low-income receiving government payments), private health insurance, and physical activity level (determined by a 5-level mobility scale and analysed as 'active' or 'sedentary'), were self-reported. Highest level of education was recorded as one of five different levels ('Primary school or less', 'Secondary education (not completed), 'Secondary education (completed),' 'Technical and Further Education (TAFE)/Trade' and 'University'). Due to small counts in the lowest education group $(n=28)$, the two lower levels of educational attainment were combined for analyses.

Alcohol consumption was determined using the Victorian Cancer Council Food Frequency Questionnaire [36] and categorised as meeting or exceeding National Health and Medical Research Council (NHMRC) of Australia guidelines of two standard drinks or less per day [37]. Height and weight were to the nearest $0.1 \mathrm{~cm}$ and $0.1 \mathrm{~kg}$, respectively. Body mass index (BMI) was calculated as (weight in $\mathrm{kg}$ )/(height in metres) ${ }^{2}$. Waist circumference (minimal abdominal) was measured using an anthropometric tape measure and categorised as $<80 \mathrm{~cm}$ or $\geq 80 \mathrm{~cm}$ as per NHMRC guidelines [38].

Area based socioeconomic status (SES) was determined using the Australian Bureau of Statistic (ABS) Index of Relative Socio-economic Advantage and Disadvantage
(IRSAD). The IRSAD is a calculation of the level of social advantage/disadvantage based on 2011 ABS census data for each ABS Census Collection District, an area that encompasses approximately 250 households. Participant residential addresses were matched with corresponding ABS collection district to determine values according to the Socio Economic Indexes For Areas (SEIFA), from which IRSAD scores were ascertained and used to categorise area-level SES into quintiles, whereby quintile 1 was the most disadvantaged and quintile 5 was the most advantaged [39].

\section{Analyses}

Missing values for HLQ items were imputed using the expectation maximisation (EM) algorithm, as previously employed by Beauchamp et al. [4]. The EM algorithm imputes values for scales where there are no more than 2 values missing from 4 to 5 item scales and no more than 3 values missing from 6 item scales.

Effect sizes (ES) were calculated using Cohen's d [40] for differences in mean HLQ scale scores between demographic groups; ES of 0.20 to $0.50,0.5$ to 0.80 and $>0.80$ were considered small, medium, and large, respectively. For all HLQ scales, assumptions of normal distribution were not met, although responses covered the full range of each scales, with modest floor and ceiling effects. A number of scales also violated homogeneity of variances. We therefore used robust analysis of variance (ANOVA) for analysis of demographic differences in HLQ scores, using the Welch method for scales that violated homogeneity of variances.

Linear regression analyses were used to investigate associations between HLQ scale scores and SES and education level. Associations between HLQ scale scores and education level and SES quintile are presented as predicted means and $p$-values, comparing the mean scale score for categories 2-4 against the mean scale score for category 1 . Post-hoc analysis was undertaken to investigate the relationship between age and alcohol intake.

Analyses were undertaken using SPSS version 22 and Minitab (version 16; Minitab, State College, PA).

\section{Results}

Of 1032 women sent the HLQ, 20 had died, 264 could not be contacted, and 35 did not participate due to reasons including illness, age, time restraints and lack of interest. Thus, 713 women provided HLQ data and were included in this analysis. Twenty-six women were assisted to complete the questionnaire over the telephone and a further 16 women were assisted by a friend or relative.

Participant characteristics are presented in Table 1. Participant SES spanned all IRSAD levels, with similar proportions observed in the most disadvantaged (14.9\%) 
Table 1 Participant characteristics $(n=713)$ given as $n(\%)$ or median (IQR)

\begin{tabular}{|c|c|c|}
\hline Demographic characteristics & $\begin{array}{l}\mathrm{n}(\%) \text { or median } \\
\text { (IQR) }\end{array}$ & missing data $n$ \\
\hline Age & $59.1(45.2-70.2)$ & 0 \\
\hline Lives alone & $138(19.8)$ & 16 \\
\hline Secondary education incomplete & $240(33.8)$ & 2 \\
\hline Education (4 levels) & & 2 \\
\hline Secondary education (incomplete) & $240(33.8)$ & \\
\hline Secondary education (complete) & $146(20.5)$ & \\
\hline TAFE/Trade & $141(19.8)$ & \\
\hline University & $184(25.9)$ & \\
\hline Private health insurance & $496(71.0)$ & 14 \\
\hline Health care concession card & $283(40.5)$ & 15 \\
\hline Born in Australia & $614(86.2)$ & 1 \\
\hline English spoken at home & $706(99.3)$ & 7 \\
\hline IRSAD Quintiles & & 37 \\
\hline 1 (most disadvantaged) & $101(14.9)$ & \\
\hline 2 & $72(10.7)$ & \\
\hline 3 & $258(38.2)$ & \\
\hline 4 & $131(19.4)$ & \\
\hline 5 (least disadvantaged) & $114(16.9)$ & \\
\hline$\geq 3$ Health conditions & $143(20.1)$ & 22 \\
\hline $\mathrm{BMI} \geq 25$ & $435(66.3)$ & 57 \\
\hline Waist circumference $\geq 80 \mathrm{~cm}$ & $464(70.5)$ & 55 \\
\hline Sedentary activity & $163(24.5)$ & 48 \\
\hline Current smoking & $69(10.4)$ & 47 \\
\hline$>2$ glasses alcohol per day & $160(24.1)$ & 48 \\
\hline
\end{tabular}

$B M I$ body mass index, IRSAD Index of Relative Socioeconomic Advantage and Disadvantage and the most advantaged (16.9\%) quintiles. Only $5(0.71 \%)$ participants reported speaking a language other than English at home and 69 (10.4\%) reported current smoking. Almost two-thirds of participants $(n=435)$ had a BMI $\geq 25 \mathrm{~kg} / \mathrm{m}^{2}$ and $464(70.5 \%)$ had a waist circumference of $\geq 80 \mathrm{~cm}$.

Mean HLQ scale scores are shown in Table 2. The highest mean score for scales 1-5 was observed for Scale 1. 'Feeling understood and supported by healthcare professionals' (mean 3.20, \pm SD 0.52) while the lowest mean score was observed for Scale 5. 'Appraisal of health information' (mean 2.81, \pm SD 0.48). Scale 9. 'Understand health information well enough to know what to do' displayed the highest score of scales 6-9 (mean 4.28, \pm SD 0.54), while Scale 7. 'Navigating the healthcare system' displayed the lowest mean score (mean 4.09, \pm SD 0.57).

Tables 3 and 4 summarise the association between sociodemographic characteristics and anthropometric and lifestyle risk-factors, and the nine HLQ scales. ES observed for differences in mean HLQ scale scores between sociodemographic groups were all small $(0.20$ to 0.50$)$. The largest ES was 0.45 , which related to differences in mean scale scores for Scale 8. 'Ability to find good health information' between age $<65$ vs $\geq 65$ years (ES $0.45,95 \%$ CI 0.40 to 0.54 ), self-reported health conditions $<3$ vs $\geq 3$ (ES $0.45,95 \%$ CI 0.41 to 0.57 ) and also sedentary vs active physical activity (ES $0.45,95 \%$ CI 0.40 to 0.56 ). Being born overseas was associated with lower mean scores in Scale 2. 'Having sufficient information to manage health' (ES 0.30, 95\% CI 0.26 to 0.38 ) and Scale 8. 'Ability to find good health information' (ES 0.26, 95\% CI 0.21 to 0.39). Having private health insurance was associated with higher mean scores in the greatest number of HLQ

Table 2 HLQ scores for each of the 9 scales $(n=712)$ given as mean with standard deviation $( \pm$ SD), and 95\% confidence interval [95\% Cl]

\begin{tabular}{|c|c|c|c|}
\hline Scale & HLQ Scale & Mean $( \pm \mathrm{SD}) \quad[95 \% \mathrm{Cl}]$ & Missing data (n) \\
\hline & & $\begin{array}{l}\text { Range } 1-4 \\
\text { (lowest - highest) }\end{array}$ & \\
\hline 1 & Feeling understood and supported by healthcare professionals & $3.20(0.52)[3.16,3.23]$ & 1 \\
\hline 2 & Having sufficient information to manage my health & $3.07(0.44)[3.04,3.11]$ & 1 \\
\hline 3 & Actively managing my health & $2.99(0.49)[2.95,3.02]$ & 3 \\
\hline 4 & Social support for health & $3.08(0.50)[3.05,3.12]$ & 2 \\
\hline \multirow[t]{2}{*}{5} & Appraisal of health information & $2.81(0.48)[2.77,2.84]$ & 3 \\
\hline & & $\begin{array}{l}\text { Range } 1-5 \\
\text { (lowest - highest) }\end{array}$ & \\
\hline 6 & Ability to actively engage with healthcare professionals & $4.17(0.58)[4.13,4.21]$ & 9 \\
\hline 7 & Navigating the healthcare system & $4.09(0.57)[4.05,4.13]$ & 10 \\
\hline 8 & Ability to find good health information & $4.11(0.59)[4.06,4.15]$ & 10 \\
\hline 9 & Understand health information well enough to know what to do & $4.28(0.54)[4.24,4.32]$ & 9 \\
\hline
\end{tabular}




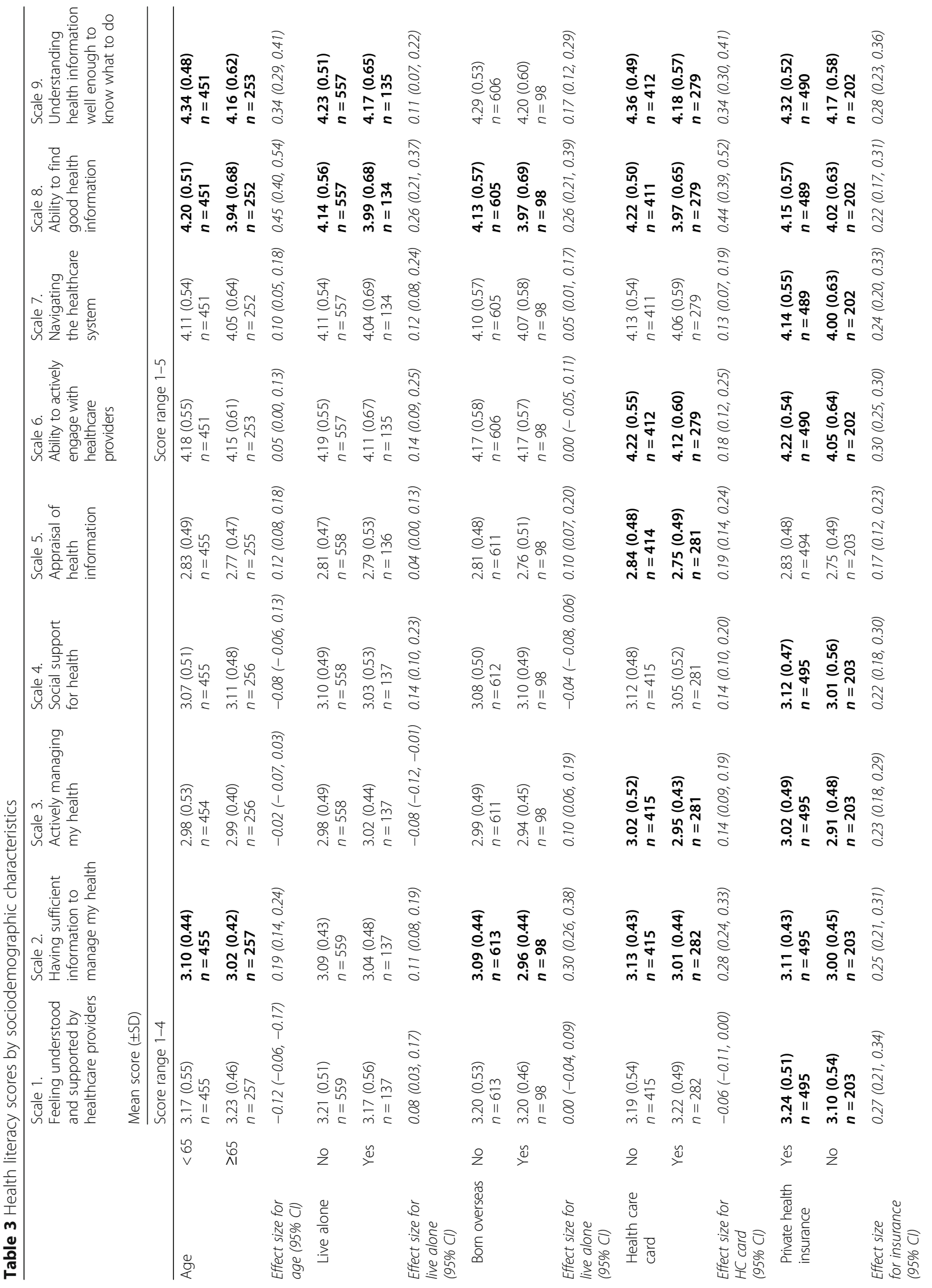




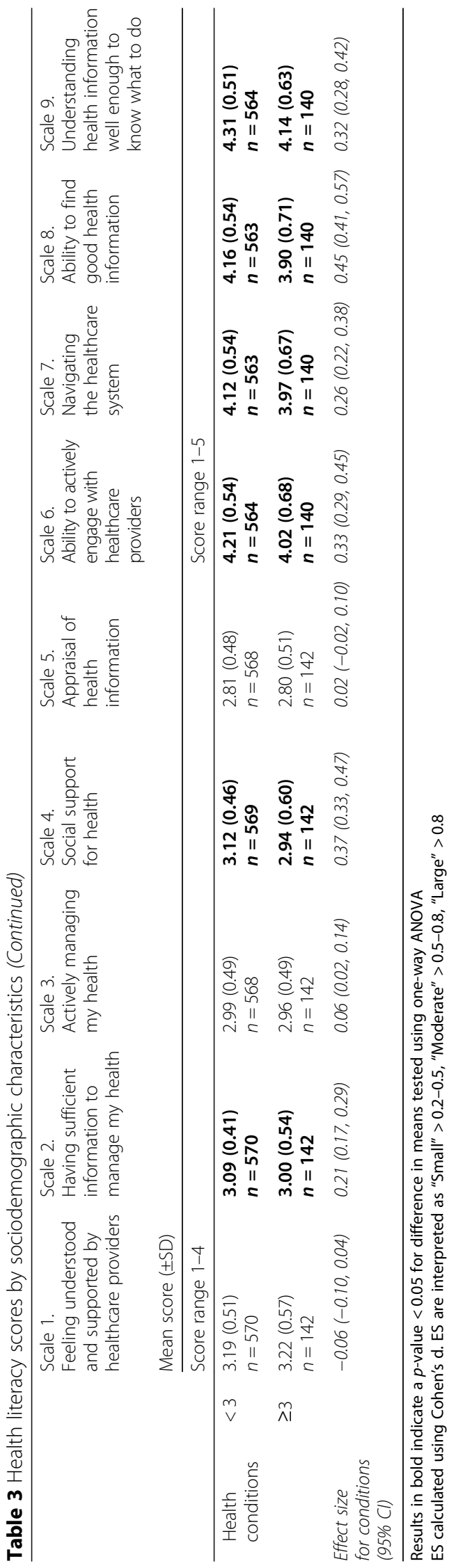




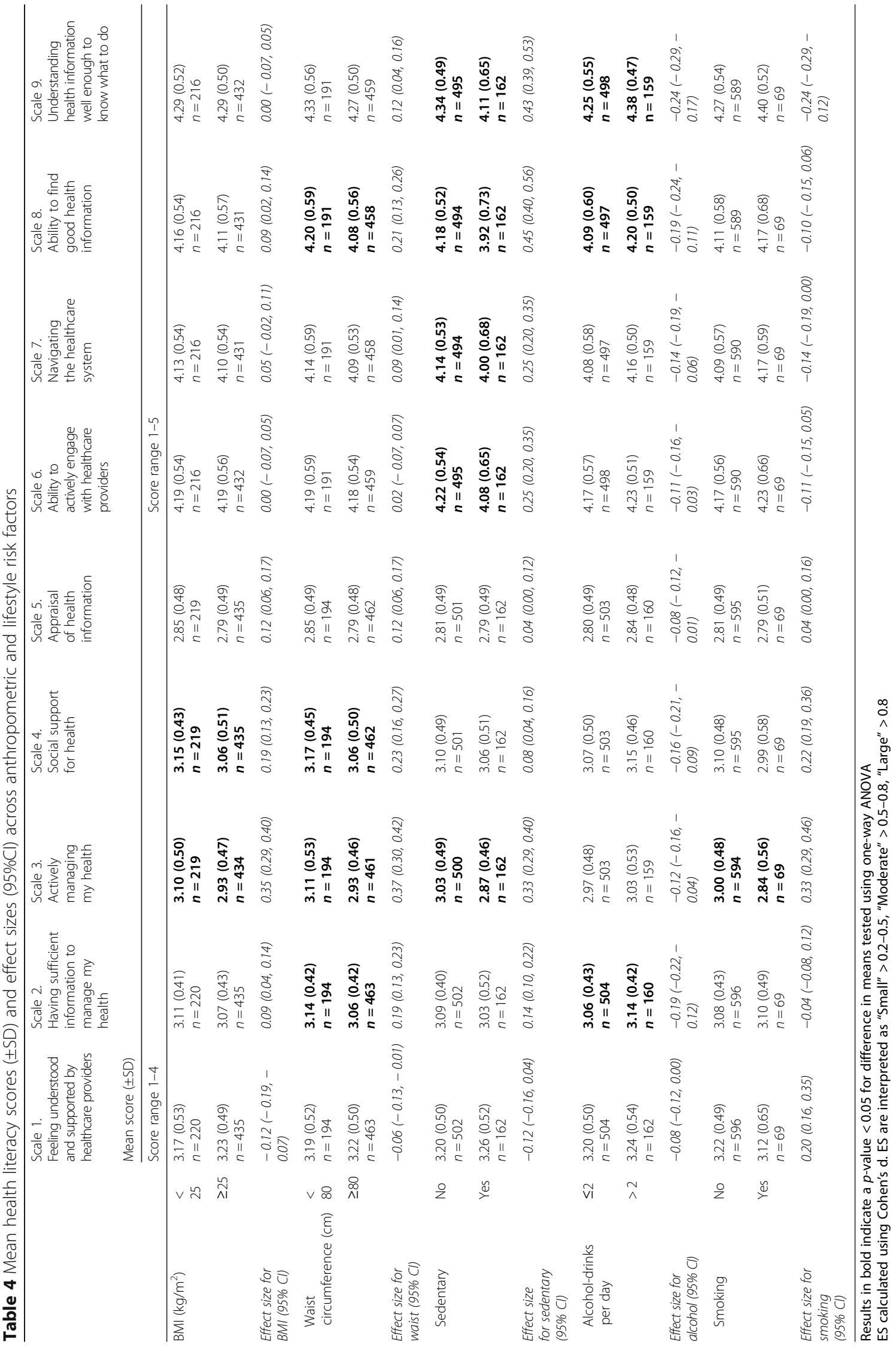


scales of any demographic characteristic, showing small but significant ES for all scales except Scale 5 . 'Appraisal of health information'. Private health insurance was also the only demographic characteristic that showed a significant ES for mean differences in Scale 1. 'Feeling understood and supported by healthcare providers' (ES $0.27,95 \%$ CI 0.21 to 0.34 ).

Figure 1 illustrates the relationship between highest self-reported education level and health literacy scales. Associations for Scale 7. 'Navigating the healthcare system' and Scale 9. 'Understand health information well enough to know what to do' were non-linear. Women who did not complete secondary education and women with a TAFE or trade qualification showed lower mean scores than individuals who self-reported their highest level of education as secondary education (complete) or a university degree. Appendix Table 6 provides $p$-values for trend across HLQ scales for both education level and SES.

Figure 2 describes the associations between area-level SES and seven of the nine health literacy scales. Scale 5 . 'Appraisal of health information' did not show any association while Scale 2. 'Having sufficient information to manage health' showed a non-linear trend $(p=0.05)$, with SES quintiles 3 and 5 showing an association with higher scale scores while holding quintile 1 as referent. All other scales showed a significant association, however, four of these associations were also non-linear.

Table 4 shows associations between lifestyle and anthropometric risk factors for chronic disease. Having a high BMI or waist circumference were both associated with lower scores in Scale 3. 'Actively managing my health' and Scale 4. 'Social support for health'. Sedentary behaviour was associated with the greatest number of health literacy scales of any lifestyle or anthropometric risk factor for chronic disease and adjusting for age, education level or SES quintile did not change associations. A high alcohol intake was associated with higher mean scores for two health literacy scales (Table 2). A post hoc analysis revealed an association between age and alcohol intake, with a greater number of women in younger age groups (including women in their 30s, 40s and 50s) more likely to consume alcohol above recommended levels than women in older age groups (data not shown).

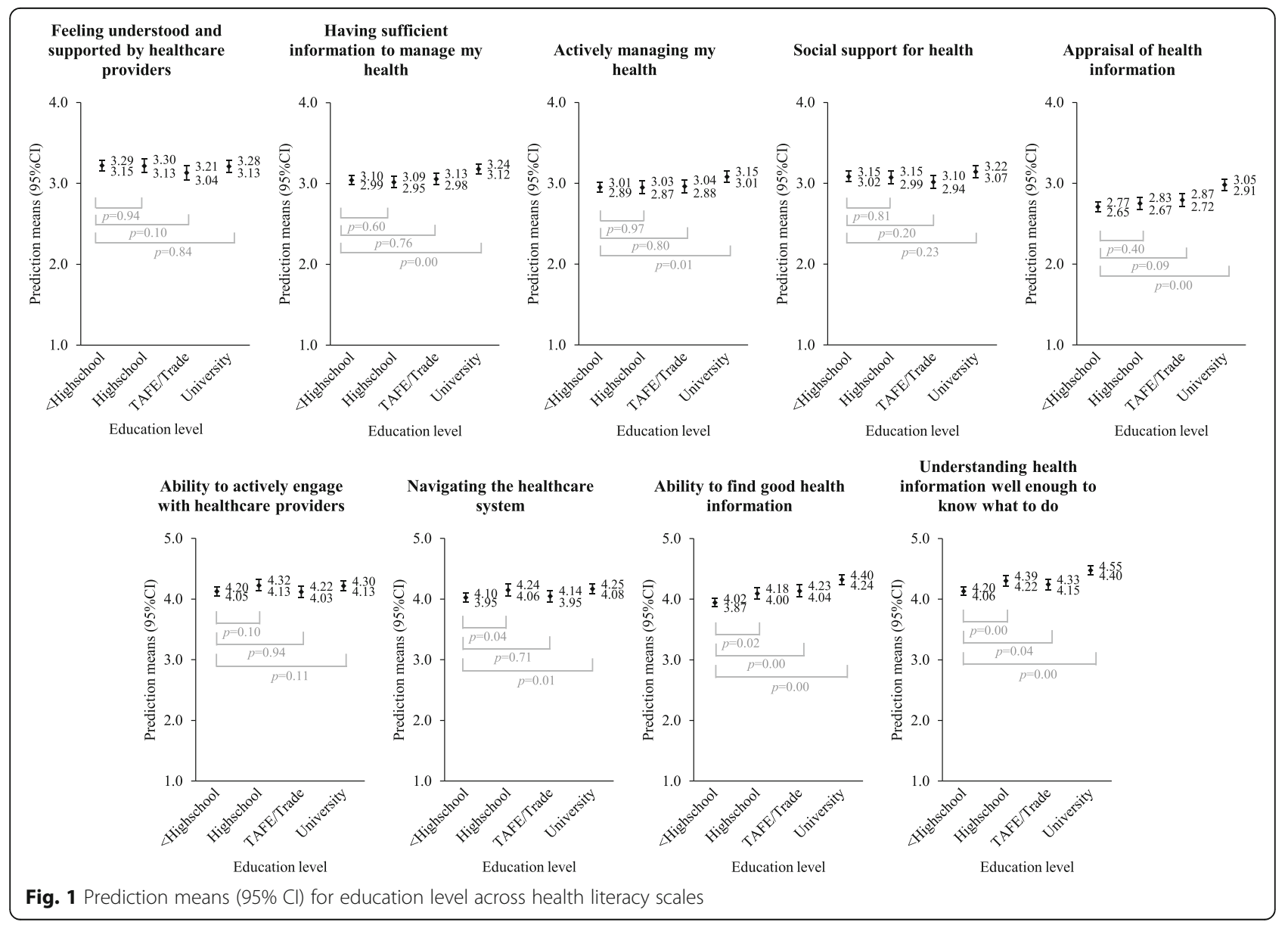




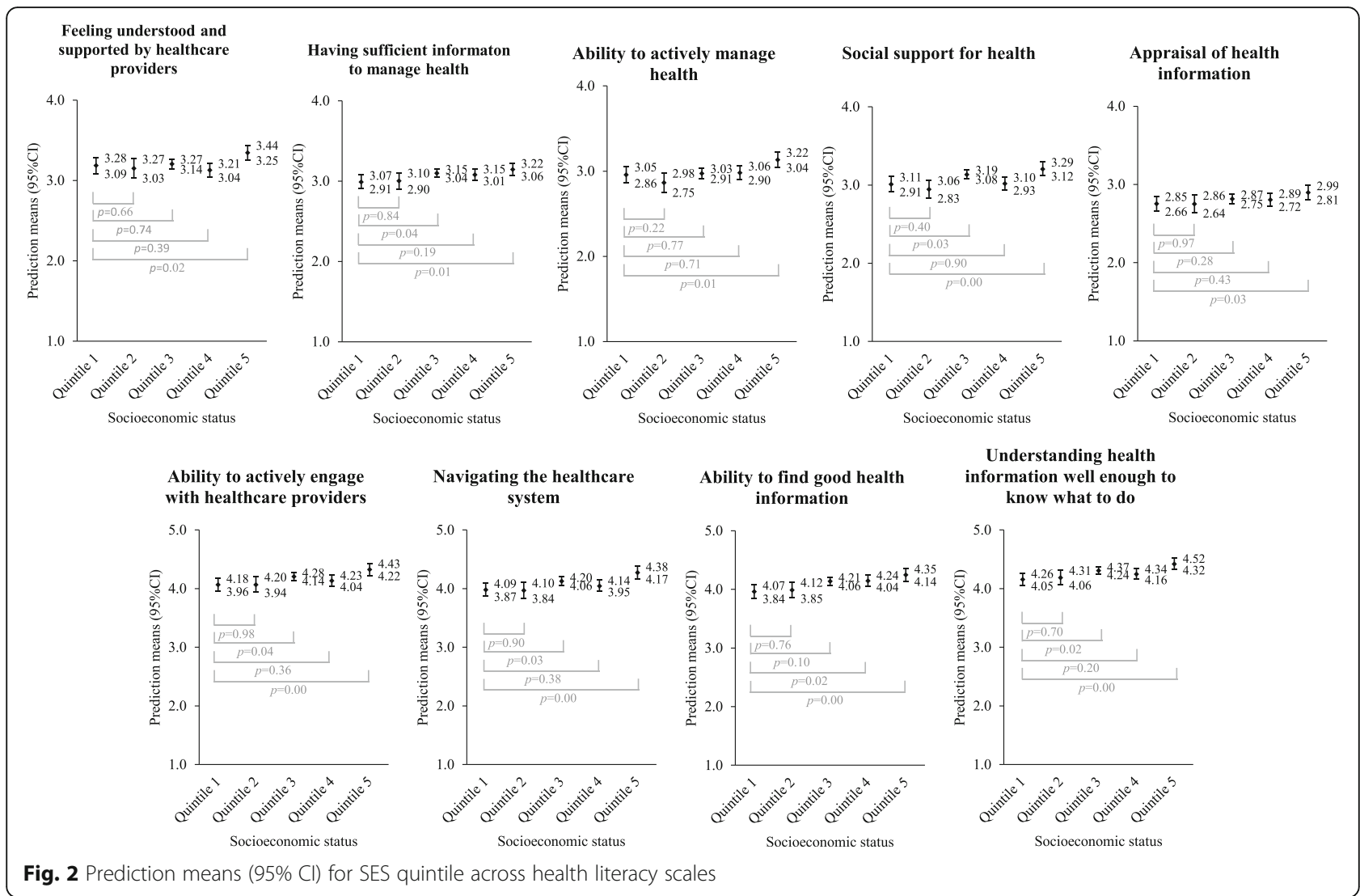

\section{Discussion}

Women within this study displayed strengths and difficulties across nine domains of health literacy with mean scores varying across the HLQ scales. Sociodemographic characteristics including older age, lower education level, lower area-level SES, country of birth other than Australia, and increasing number of chronic health conditions were all associated with lower health literacy. However, regression analyses revealed associations that appeared non-linear, between some HLQ scales and education level and SES quintile.

\section{Sociodemographic characteristics}

Age and country of birth have previously shown strong associations with multiple scales of the HLQ in Australian study populations $[9,10,41]$. However, our current study showed smaller ES and associations in fewer health literacy scales for both of these sociodemographic characteristics. Specific to country of birth, it is possible that language barriers were driving the effect seen between health literacy and country of birth in earlier studies, whilst the small proportion of participants in our study that did not speak English at home (0.7\%) may explain why our results differed.
Previous research has revealed inconsistent associations between health literacy and social advantage and/ or disadvantage. Associations between HLQ scale scores and SES and education vary between studies, possibly related to differences in the definition or measurement of parameters of social advantage/disadvantage, for instance income, occupation, highest level of education, or having private health insurance [5, 6]. Completion of secondary education and greater number of years in education have both previously been associated with higher HLQ scores [4-6]. Our study found a similar relationship; however, we observed a non-linear relationship, with similar health literacy scores observed for the 'TAFE/Trade' group and the 'Secondary education incomplete' category. This suggests that education type, in addition to time spent in formal education or completion of secondary education, may be important to health literacy. This speculation is supported by data from university students who undertook health-based degrees and showed varying HLQ scores across the different degrees [28]. Despite the fact that all participants in that study were attending university and were therefore more likely to have higher health literacy overall, the type of degree studied was still associated with HLQ scores 
with the highest HLQ scores observed for medical students and the lowest for nursing students [28].

The appearance of non-linear associations with area-level SES in our study may potentially be due to the use of SES quintiles derived from IRSAD data, which provide a greater level of detail compared to other studies that employed, for instance, 2- or 3-level measures of income [5, 6]. Given that education and income variables form part of the aggregate IRSAD values, it may also be that education level, more so than income or other indicators of advantage/disadvantage, are underpinning these non-linear associations. This seems particularly likely in light of the well-documented interconnectedness between education and income, and the inextricable link between education, income and health literacy.

\section{Anthropometric and lifestyle risk factors}

Previous research has shown a greater likelihood of lower health literacy in individuals with a chronic health condition $[4,30,42]$. This may be explained by higher health literacy needs of individuals managing a chronic disease, or low health literacy leading to chronic illness, or both. We found associations between HLQ scores and anthropometric and lifestyle risk factors known to be associated with chronic diseases, suggesting that health literacy may play a mediating role in the development of chronic disease.

The exception to this was the association between higher HLQ scores and alcohol intake above recommended levels. These associations were seen for the same HLQ scales which displayed an inverse association with age. Thus, we undertook a post-hoc analysis to determine whether age was inversely associated with alcohol intake and, in keeping with previous research [43], we observed that younger women were more likely to exceed recommendations for alcohol intake. Together these results indicate that associations between higher HLQ scores and alcohol intake above recommended levels are likely driven by age. These results are similar to a previous study of Danish adults with diabetes in which no associations were seen between alcohol consumption and the two HLQ scales assessed, Scale 9. 'Understanding health information well enough to know what to do' and Scale 6. 'Ability to actively engage with healthcare providers', after adjusting for sociodemographic characteristics, including age [12].

While, associations between levels of physical activity and HLQ scores have been seen in a small study of 36 women diagnosed with breast cancer [13], and in a large $(n=29,473)$ population-based study of Danish adults with diabetes [12], HLQ scales associated with physical activity differed across those two studies and also our current study. These differences may be due to heterogeneous study populations and use of different measures of physical activity.

\section{Strengths and limitations}

Our study has a number of strengths. We utilised a population-based sample of women and a multidimensional measure of health literacy that enabled us to examine associations between specific aspects of health literacy and sociodemographic, anthropometric and lifestyle characteristics. The use of objective measures such as BMI and waist circumference are also a strength of this study.

A possible limitation of the study could be the underrepresentation of women with low health literacy due to the requirements of participation including the ability to read and understand the invitation to participate, complete questionnaires and attend clinical appointments. To mitigate this bias, we made efforts to offer assistance for completing questionnaires if needed. Similar to previous studies, we also avoided use of the term 'health literacy' in all communications to avoid the possibility that women with low literacy may have refused participation due to stigma or shame [33]. Data regarding smoking, physical activity and alcohol consumption were self-reported and are also subject to potential bias. Our current study was undertaken within a geographically defined area of regional Victoria, and thus results may not be generalisable to the wider Australian female population. Finally, this study focused on a cohort of Australian women. Future research is required to investigate whether similar associations exist in a population-based cohort of Australian men.

\section{Conclusion}

We used a multidimensional health literacy tool to describe the health literacy profile of a randomly selected, population-based sample of Australian women and investigate associations between health literacy and sociodemographic, anthropometric and lifestyle characteristics. Mean scores varied across the nine HLQ scales indicating women in this study have strengths and difficulties in different aspects of health literacy. We report associations between lower health literacy and sociodemographic characteristics including lower SES, lower levels of education, and having been born overseas. Unlike previous studies, the associations we observed between health literacy and education and SES were non-linear, potentially due to the different measures of education and SES used. We also demonstrated associations between low health literacy and anthropometric and lifestyle risk factors for chronic disease. Further research in large population-based studies, using robust measures of lifestyle risk factors is required to better understand the relationship between lifestyle management of health and health literacy. 


\section{Appendix}

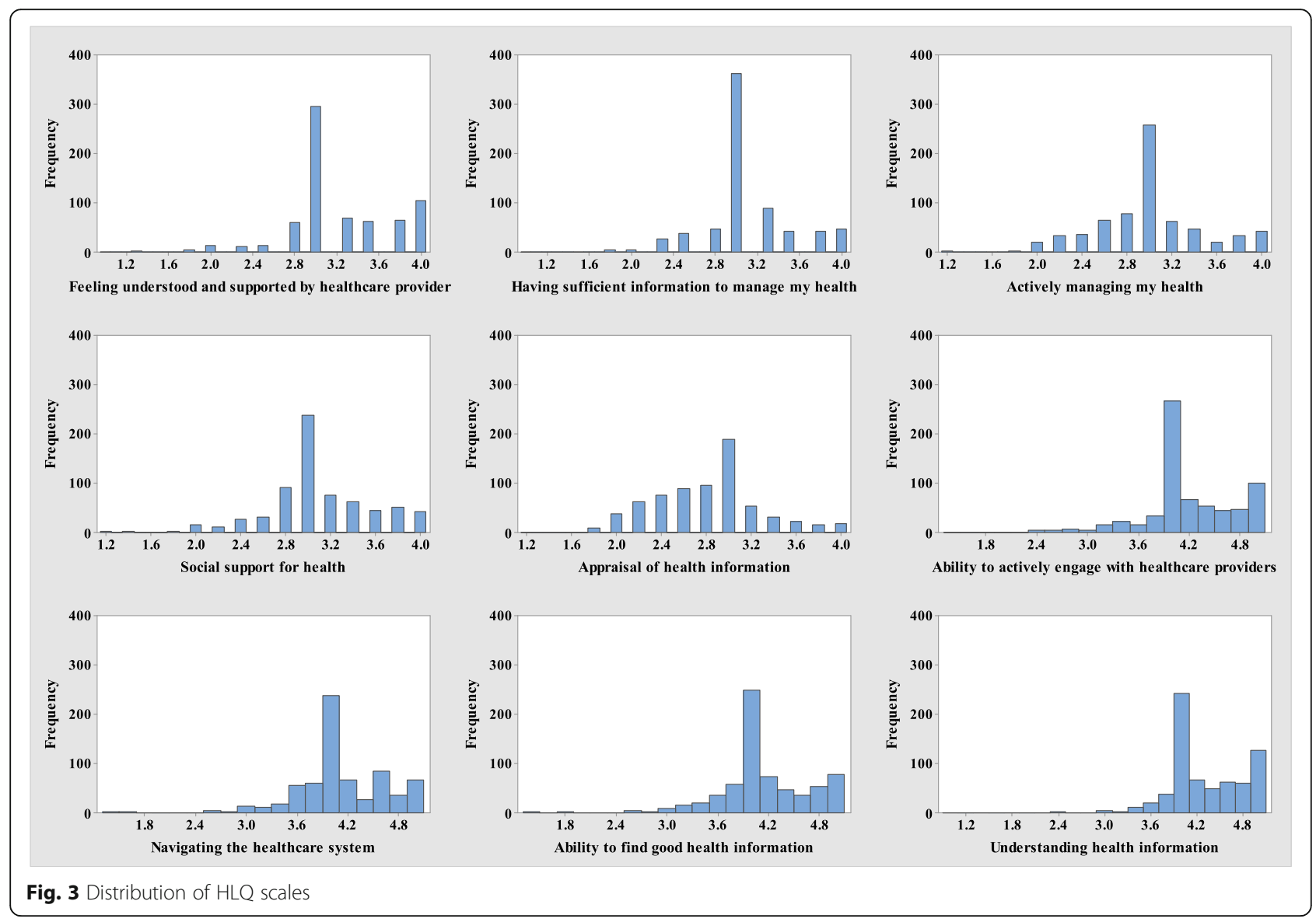




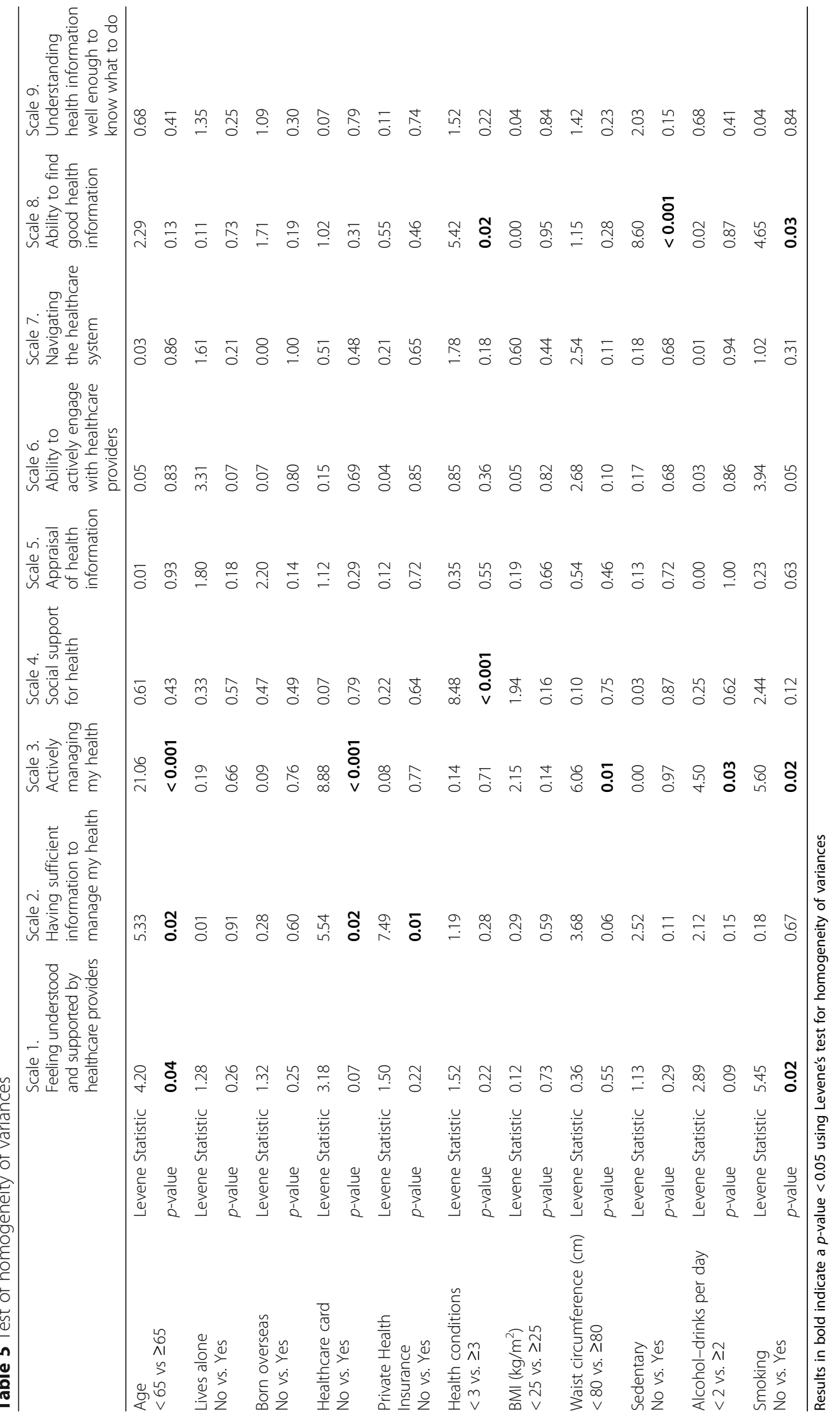


Table 6 Test for trend between education level and SES and HLQ scales

\begin{tabular}{lll}
\hline HLQ Scale & Education $p$-value & SES $p$-value \\
\hline Feeling understood and supported by healthcare providers & 0.37 & $\mathbf{0 . 0 1}$ \\
Having sufficient information to manage my health & $<\mathbf{0 . 0 0 1}$ & 0.05 \\
Actively managing my health & $\mathbf{0 . 0 3}$ & $<\mathbf{0 . 0 0 1}$ \\
Social support for health & 0.15 & $<\mathbf{0 . 0 0 1}$ \\
Appraisal of health information & $<\mathbf{0 . 0 0 1}$ & 0.19 \\
Ability to actively engage with healthcare providers & 0.18 & $\mathbf{0 . 0 0 1}$ \\
Navigating the healthcare system & $\mathbf{0 . 0 4}$ & $<\mathbf{0 0 1}$ \\
Ability to find good health information & $<\mathbf{0 . 0 0 1}$ & $<\mathbf{0 . 0 0 1}$ \\
Understanding health information well enough to know what to do & $<\mathbf{0 . 0 0 1}$ & $<\mathbf{0 . 0 0 1}$ \\
\hline
\end{tabular}

Results in bold indicate a $p$-value $<0.05$

\section{Abbreviations}

ABS: Australian Bureau of Statistics; ANOVA: Analysis of Variance; BMl: Body Mass Index; EM: Expectation Maximisation; ES: Effect Size; GOS: Geelong Osteoporosis Study; HLQ: Health Literacy Questionnaire; IRSAD: Index of Relative Socioeconomic Advantage and Disadvantage; NHMRC: National Health and Medical Research Council; REDCap: Research Electronic Data Capture; SD: Standard Deviation; SEIFA: Socio Economic Indexes For Areas; SES: Socioeconomic Status; WHO: World Health Organization

\section{Acknowledgments}

The authors would like to acknowledge the contributions of the research staff and participants of the Geelong Osteoporosis Study.

\section{Funding}

The Geelong Osteoporosis Study is funded by the National Health and Medical Research Council (NHMRC) of Australia (Project number 628582). SLB-O and LJW are supported by NHMRC Career Development Fellowships (1107510, and 1064272, respectively). RB is supported by an NHMRC Senior Principal Research Fellowship (1082138).

\section{Availability of data and materials}

The datasets analysed for the current study are available from the author Julie Pasco on reasonable request.

\section{Authors' contributions}

Author SMH led the data collection, analysis and preparation of drafts. Authors SLB-O, JAP, LWW and RB contributed to the initial conception of the study and provided ongoing guidance in facilitating the research. $A B$ provided guidance for the data analysis strategy and JAP and SLB-O contributed to interpretation of results and initial drafts. All authors contributed to second and subsequent drafts and approved the final draft.

\section{Ethics approval and consent to participate}

This research project was approved by the Barwon Health Human Research Ethics Committee (HREC) (Reference no. 92/01_E7). All participants gave written informed consent for participation.

\section{Consent to publication}

Not applicable

\section{Competing interests}

The authors declare that they have no competing interests.

\section{Publisher's Note}

Springer Nature remains neutral with regard to jurisdictional claims in published maps and institutional affiliations.

\section{Author details}

${ }^{1}$ Epi-Centre for Healthy Ageing, School of Medicine, IMPACT SRC, Deakin University, PO Box 281 (Barwon Health), Geelong, VIC 3220, Australia.

${ }^{2}$ Australian Institute for Musculoskeletal Science (AIMSS), The University of
Melbourne and Western Health, St Albans, VIC, Australia. ${ }^{3}$ Centre for Medicine Use and Safety, Faculty of Pharmacy and Pharmaceutical Sciences, Monash University, Parkville, VIC, Australia. ${ }^{4}$ Institute for Health and Ageing, Australian Catholic University, Melbourne, VIC, Australia. ${ }^{5}$ Department of Medicine-Western Health, Melbourne Medical School, The University of Melbourne, St Albans, VIC, Australia. ${ }^{6}$ Australian Health Policy Collaboration, Melbourne, VIC, Australia. ${ }^{7}$ Department of Rural Health, Monash University, Moe, VIC, Australia. ${ }^{8}$ Department of Epidemiology and Preventive Medicine, School of Public Health and Preventive Medicine, Monash University, Melbourne, VIC, Australia. ${ }^{9}$ Monash Department of Clinical Epidemiology, Cabrini Institute, Malvern, VIC, Australia.

Received: 24 October 2017 Accepted: 26 June 2018

Published online: 13 July 2018

\section{References}

1. World Health Organization. Health promotion glossary. Health Promot Int 1998;13:349-64.

2. Australian Bureau of Statistics. Adult Literacy and Life Skills Survey (ALLS). 2009.

3. OECD Statistics Canada. Literacy for life: further results from the adult literacy and life skills survey second international ALL report. OECD publishing; 2011.

4. Beauchamp A, Buchbinder R, Dodson S, Batterham RW, Elsworth GR, McPhee $C$, et al. Distribution of health literacy strengths and weaknesses across sociodemographic groups: a cross-sectional survey using the health literacy questionnaire (HLQ). BMC Public Health BMC Public Health. 2015:15:678,

5. Jessup RL, Osborne RH, Beauchamp A, Bourne A, Buchbinder R. Health literacy of recently hospitalised patients: a cross-sectional survey using the health literacy questionnaire (HLQ). BMC Heal Serv Res BMC Health Services Research. 2017:17:52

6. Bo A, Friis K, Osborne RH, Maindal HT. National indicators of health literacy: ability to understand health information and to engage actively with healthcare providers - a population-based survey among Danish adults. BMC Public Health. 2014;14:1095.

7. Howard DH, Sentell T, Gazmararian JA. Impact of health literacy on socioeconomic and racial differences in health in an elderly population. J Gen Intern Med. 2006:21:857-61.

8. Friis K, Lasgaard M, Rowlands G, Osborne RH, Maindal HT. Health literacy mediates the relationship between educational attainment and health behavior: a Danish population-based study. J Health Commun Routledge. 2016:21:54-60.

9. Berkman ND, Sheridan SL, Donahue KE, Halpern DJ, Crotty K. Low Health Literacy and Health Outcomes: An updated systematic review. Ann Intern Med. American College of Physicians. 2011;155:97-W41.

10. Mackey LM, Doody C, Werner EL, Fullen B. Self-management skills in chronic disease management: what role does health literacy have? Med Decis Mak. 2016:36:741-59.

11. Demian MN, Shapiro RJ, Thornton WL. An observational study of health literacy and medication adherence in adult kidney transplant recipients. Clin Kidney J. 2016;9:858-65. 
12. Friis $K$, Vind BD, Simmons RK, Maindal HT. The relationship between health literacy and health behaviour in people with diabetes: a Danish populationbased study. J Diabetes Res. 2016;2016:1-7.

13. Plummer LC, Chalmers KA. Health literacy and physical activity in women diagnosed with breast cancer. Psycho-oncology. 2016;26:1478-83.

14. Wolf MS, Gazmararian JA, Baker DW. Health literacy and health risk behaviors among older adults. Am J Prev Med. 2007;32:19-24.

15. Kim S, Love F, Quistberg DA, Shea JA. Association of health literacy with self-management behavior in patients with diabetes. Diabetes Care. 2004;27:2980-2

16. von Wagner C, Knight K, Steptoe A, Wardle J. Functional health literacy and health-promoting behaviour in a national sample of British adults. J Epidemiol Community Heal. 2007;61:1086-90.

17. Carbone ET, Zoellner JM. Nutrition and health literacy: a systematic review to inform nutrition research and practice. J Acad Nutr Diet Elsevier. 2012;112:254-65.

18. Tokuda Y, Doba N, Butler JP, Paasche-Orlow MK. Health literacy and physical and psychological wellbeing in Japanese adults. Patient Educ Couns. 2009;75:411-7.

19. Wolf MS, Gazmararian JA, Baker DW. Health literacy and functional health status among older adults. Arch Intern Med. 2005;165:1946-52.

20. Jordan JE, Osborne RH, Buchbinder R. Critical appraisal of health literacy indices revealed variable underlying constructs, narrow content and psychometric weaknesses. J Clin Epidemiol. 2011;64:366-79.

21. Pleasant A, McKinney J, Rikard RV. Health literacy measurement: a proposed research agenda. J Health Commun Taylor \& Francis. 2011;16:11-21.

22. Haun JN, Valerio MA, McCormack LA, Sørensen K, Paasche-Orlow MK. Health literacy measurement: an inventory and descriptive summary of 51 instruments. J Health Commun. 2014;19:302-33.

23. McCormack L, Haun J, Sørensen K, Valerio M. Recommendations for advancing health literacy measurement. J Health Commun. 2013;18:9-14.

24. Osborne RH, Batterham RW, Elsworth GR, Hawkins M, Buchbinder R. The grounded psychometric development and initial validation of the health literacy questionnaire (HLQ). BMC public health. BioMed Central. 2013;13:658

25. Sørensen K, Van den BS, Pelikan JM, Fullam J, Doyle G, Slonska Z, et al. Measuring health literacy in populations: illuminating the design anddevelopment process of the European health literacy survey questionnaire(HLS-EU-Q). BMC Public Health. 2013;13:948.

26. Australian Bureau of Statistics. Health literacy - Adult Litearcy and Life Skills Survey. 2009, cat. no. 4102.0, ABS, Canberra, Australia.

27. Batterham RW, Hawkins M, Collins PA, Buchbinder R, Osborne RH. Health literacy: applying current concepts to improve health services and reduce health inequalities. Public Health. 2016;132:3-12.

28. Mullan J, Burns P, Weston K, Mclennan P, Rich W, Crowther S, et al. Health literacy amongst health professional university Students : a study using the Health Literacy Questionnaire. Educ Sci. 2017;7:1-11.

29. Zhang Y, Zhang F, Hu P, Huang W, Lu L, Bai R, et al. Exploring health literacy in medical university students of Chongqing, China: a crosssectional study. PLoS One. 2016;11:e0152547.

30. Goeman D, Conway S, Norman R, Morley J, Weerasuriya R, Osborne RH, et al. Optimising health literacy and access of service provision to community dwelling older people with diabetes receiving home nursing support. J Diabetes Res. 2016;2016:1-12.

31. Buchbinder R, Batterham R, Cicirello S, Newman S, Horgan B, Ueffing E, et al. Health literacy: what is it and why is it important to measure? J Rheumatol. 2011:38:1791-7.

32. Morris RL, Soh S-E, Hill KD, Buchbinder R, Lowthian JA, Redfern J, et al. Measurement properties of the health literacy questionnaire $(\mathrm{HLQ})$ among older adults who present to the emergency department after a fall: a Rasch analysis. BMC Health Serv Res BMC Health Services Research. 2017;17:605.

33. Beauchamp A, Batterham RW, Dodson S, Astbury B, Elsworth GR, McPhee C, et al. Systematic development and implementation of interventions to OPtimise health literacy and access (Ophelia). BMC Public Health. BMC Public Health. 2017;17:230

34. Pasco JA, Nicholson GC, Kotowicz MA. Cohort profile: Geelong osteoporosis study. Int J Epidemiol. 2012;41:1565-75.

35. Harris PA, Taylor R, Thielke R, Payne J, Gonzalez N, Conde JG. Research electronic data capture (REDCap)-a metadata-driven methodology and workflow process for providing translational research informatics support. J Biomed Inform Elsevier Inc. 2009;42:377-81.

36. Giles GG, Ireland PD. Dietary Questionnaire for Epidemiological Studies (Version 2), Melbourne: The Cancer Council Victoria; 1996.
37. National Health and Medical Research Council. Australian Guidelines to Reduce Health Risks from Drinking Alcohol. Australian Government; 2009. Available from: www.nhmrc.gov.au/health-topics/alcohol-guidelines .

38. National Health and Medical Research Council. Clinical practice guidelines for the Management of Overweight and Obesity in adults, adolescents and children in Australia. Australian Government; 2013. Available from https:// www.nhmrc.gov.au/guidelines-publications/n57.

39. Brennan SL, Henry MJ, Wluka AE, Nicholson GC, Kotowicz MA, Pasco JA. Socioeconomic status and bone mineral density in a population-based sample of men. Bone. 2010;46:993-9.

40. Cohen J. Statistical power analysis for behavioral sciences. Revised ed. New York: Academic; 1977.

41. van der Gaag M, van der Heide I, Spreeuwenberg PMM, Brabers AEM, Rademakers JJDJM. Health literacy and primary health care use of ethnic minorities in the Netherlands. BMC Health Serv Res. BMC Health Services Research. 2017;17:350.

42. Friis K, Lasgaard M, Osborne RH, Maindal HT. Gaps in understanding health and engagement with healthcare providers across common long-term conditions: a population survey of health literacy in 29473 Danish citizens. BMJ Open. 2016:6:e009627.

43. Livingston M, Raninen J, Slade T, Swift W, Lloyd B, Dietze P. Understanding trends in Australian alcohol consumption-an age-periodcohort model. Addiction. 2016;111:1590-8.

\section{Ready to submit your research? Choose BMC and benefit from:}

- fast, convenient online submission

- thorough peer review by experienced researchers in your field

- rapid publication on acceptance

- support for research data, including large and complex data types

- gold Open Access which fosters wider collaboration and increased citations

- maximum visibility for your research: over $100 \mathrm{M}$ website views per year

At BMC, research is always in progress.

Learn more biomedcentral.com/submissions 